EPJ Web of Conferences 19, 06004 (2012)

DOI: $10.1051 /$ epjconf/20121906004

(C) Owned by the authors, published by EDP Sciences, 2012

\title{
Towards understanding the dynamics of the bar/bulge region in our Galaxy
}

\section{E. Athanassoula ${ }^{\mathrm{a}}$}

\author{
Laboratoire d'Astrophysique de Marseille (LAM), UMR6110, CNRS/Université de Provence, \\ Technopôle de Marseille Etoile, 38 rue Frédéric Joliot Curie, 13388 Marseille Cedex 20, \\ France
}

\begin{abstract}
I review some of the work on bars which is closely linked to the bar/bulge system in our Galaxy. Several independent studies, using totally independent methods, come to the same results about the 3D structure of a bar, i.e., that a bar is composed of a vertically thick inner part and a vertically thin outer part. I give examples of this from simulations and substantiate the discussion with input from orbital structure analysis and from observations. The thick part has a considerably shorter radial extent than the thin part. I then see how this applies to our Galaxy, where two bars have been reported, the COBE/DIRBE bar and the Long bar. Comparing their extents and making the reasonable and necessary assumption that our Galaxy has properties similar to those of other galaxies of similar type, leads to the conclusion that these two bars can not form a standard double bar system. I then discuss arguments in favour of the two bars being simply different parts of the same bar, the COBE/DIRBE bar being the thick inner part and the Long bar being the thin outer part of this bar. I also very briefly discuss some related new results. I first consider bar formation and evolution in disc galaxies with a gaseous component - including star formation, feedback and evolution - and a triaxial halo. Then I consider bar formation in a fully cosmological context using hydrodynamical LCDM simulations, where the host galaxies grow, accrete matter and significantly evolve during the formation and evolution of the bar.
\end{abstract}

\section{INTRODUCTION}

Bars are present in roughly two thirds of disc galaxies in the local Universe, so that the fact that our Galaxy is also barred is not exceptional. Bars have also been observed in galaxies at higher redshifts and the fraction of disc galaxies having a bar decreases with increasing redshift ([1] and references therein).

In this talk, I will will briefly review some of the theoretical results on bars which are relevant to our Galaxy and and apply them to get a better understanding of the Milky Way bar/bulge system. I will also make comparisons with observational results from external galaxies. The latter is not always straightforward, because both the viewing angle and the type of information one can obtain in these two cases are considerably different. We are located within the disc of our Galaxy, so that we have to rely on our experience with face-on external galaxies for the face-on view. Furthermore, dust obscuration is a major problem, particularly in directions near to that of the Galactic centre. On the other hand, it is possible to observe in the Milky Way individual stars in many directions and to obtain information on their metallicity and line-of-sight velocity, which is, in general, still not possible for galaxies outside our local group. In this context, the most relevant dynamical studies are those concerning the vertical bar instabilities, the corresponding orbital structure and the growth of boxy/peanut bulges (hereafter $\mathrm{b} / \mathrm{p}$, or b/p bulges).

\footnotetext{
a e-mail: lia@oamp.fr
}

This is an Open Access article distributed under the terms of the Creative Commons Attribution-Noncommercial License 3.0, which permits unrestricted use, distribution, and reproduction in any noncommercial medium, provided the original work is properly cited. 

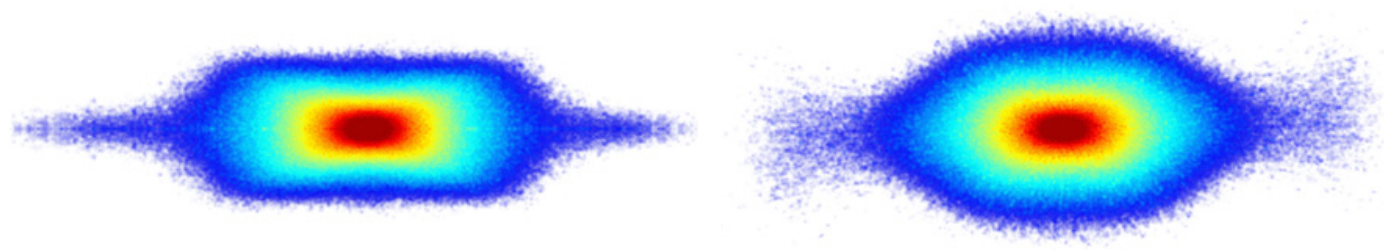

Figure 1. Side-on (left) and face-on (right) views of the bar component in a simulation. The side-on view shows clearly the boxy bulge (which is part of the bar) and the thin outer parts of the bar. The right panel view shows their face-on shape. These projections are produced with the help of the GLNEMO software (http://projets.oamp.fr/projects/glnemo2) and thus both are in perspective and can not be used to calculate length ratios directly. See text for definition of the bar component.

I will also discuss two more bar-related studies. The first one concerns some recent results on the effect of gas and of triaxial haloes on the formation and properties of the bar and peanut and the corresponding halo evolution. This work was done in collaboration with Rubens Machado and Sergey Rodionov. The second one concerns the formation of bars in fully cosmological hydrodynamical simulations, including comparisons of the resulting bar properties to both those of dynamical (idealised) simulations and those of observed bars. This work was done in collaboration with Cecilia Scannapieco.

\section{THE 3D STRUCTURE OF BARS}

Bars have a complex 3D structure. This is easy to see using N-body simulations, where one can adopt any viewing angle one wishes. I will therefore use such simulations to introduce and describe bar structure and morphology. An example of an N-body bar seen side-on ${ }^{1}$ and face-on is shown in Fig. 1. I display here only the bar component, neglecting the halo, the remaining disc and the ring which were present in this simulation. It is of course always somewhat arbitrary to decide which component a given particle belongs to, because all particles move in the same potential, that of the Galaxy. My picking out only the bar particles is therefore also somewhat arbitrary and I could have picked out more particles than I should have had, or, more likely the case here, neglected some particles which I could have included. Nevertheless, Fig. 1 shows very clearly the complex 3D structure of the bar. Over a considerable fraction, but not all, of its extent, the bar is vertically thick, much thicker than the underlying disc. Its outer parts, however, are quite thin. The face-on view shows clearly that the thick b/p component and the thin outer parts have the same position angle ${ }^{2}$ and that the latter, at least in this example, has the form of handles (ansae). It also shows clearly that the thick and the thin parts of the bar have different radial extents, as has been already noted in a number of simulations (see [2] for a summary and discussion).

A more quantitative measure of the difference between the bar and peanut lengths is given in Fig. 2. Here I show the disc component of an N-body simulation, or to be more precise, its bar region, after the bar has grown. From the upper panel (face-on view) it is possible to get a rough estimate of the bar length and from the bottom panel (side-on view) a rough estimate of the $\mathrm{b} / \mathrm{p}$ length. They are indicated by a solid and a dashed vertical line, respectively. Their location shows clearly that the bar is considerably more extended than the $\mathrm{b} / \mathrm{p}$ bulge. Let us now see how this clear, albeit complex, picture compares with the results from orbital structure and from observations.

In the simple 2D picture, the building blocks for bars are provided by a family of periodic orbits, elongated along the bar and called $x_{1}([3,4])$. In $3 \mathrm{D}$, however, the structure is more complex and the

\footnotetext{
${ }^{1}$ In a side-on view the galaxy is seen edge-on and the line of sight is along the bar minor axis.

2 I define here the bar position angle in our Galaxy as the angle between the bar major axis and the line from the centre of the Galaxy to the Sun.
} 


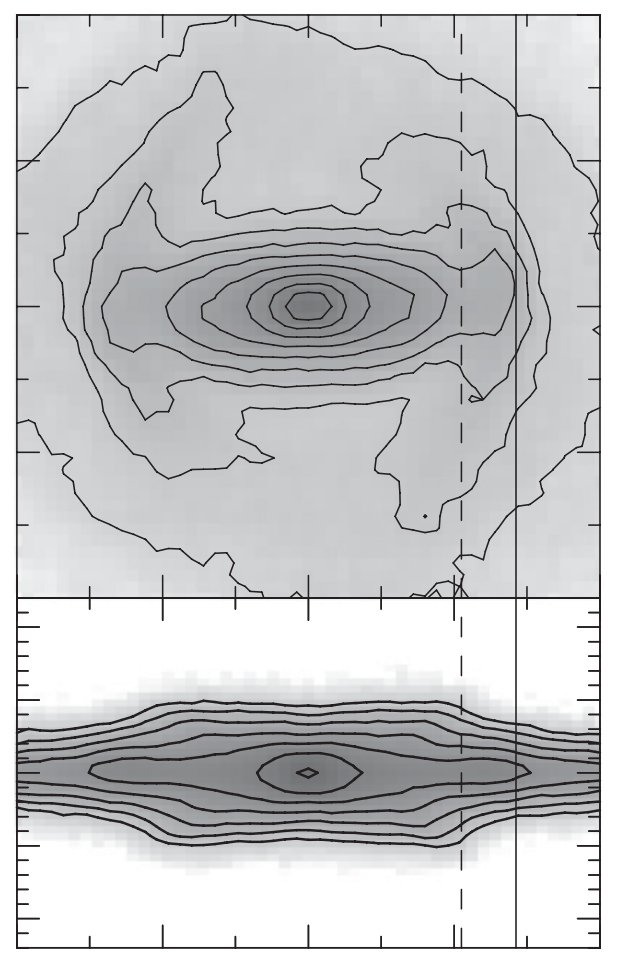

Figure 2. Face-on (top) and side-on (bottom) views of all disc particles in a N-body simulation having formed a strong bar. The solid and dashed vertical lines give estimates of the bar length and of the peanut length, respectively. The projection, contrary to Fig. 1, is orthogonal.

building blocks of this bar and of its boxy/peanut thick part come from a tree of $2 \mathrm{D}$ families as well as from $3 \mathrm{D}$ families bifurcating from the $x_{1}$ family $([5,6])$. However, it is not the same family that provides building blocks both for the bar and the b/p. The $x_{1}$ orbits are the backbone of the bar, while one or more of the vertical families provide building blocks for the $\mathrm{b} / \mathrm{p}$. The extent along the bar major axis is different in the two cases and orbital structure theory shows that the extent of the family providing building blocks for the $\mathrm{b} / \mathrm{p}$ is considerably shorter than that of the family producing the bar. In other words, the b/p should be considerably shorter than the bar, the ratio of the two extents depending on which of the vertical families is the crucial contributor to the b/p (see [7], where a number of examples and length ratios are given), in good agreement with what $\mathrm{N}$-body simulations show.

Photometry of edge-on galaxies including cuts along their major axis, as well as parallel and offset from it $([8,9])$ also argue in favour of a b/p which is shorter than the bar. This bar structure has also been studied in galaxies not edge-on but not far from it (e.g. [10-12]).

Thus all three approaches - orbital structure, N-body simulations and observations - argue cohesively that the length of the $b / p$ is less than that of the bar. I will now discuss how this can influence our view of the Milky Way's bar/bulge system.

\section{THE MILKY WAY BAR/BULGE SYSTEM}

Considerable amount of evidence has accumulated since the 1990s to argue that our Galaxy is barred (e.g. [13-20]). This bar, seen clearly in the NIR images, is often referred to as the COBE/DIRBE bar. More recently, further evidence coming from star counts at larger longitudes opened the possibility for a second bar, longer than the first one and at a larger position angle (e.g. [21-26]). 


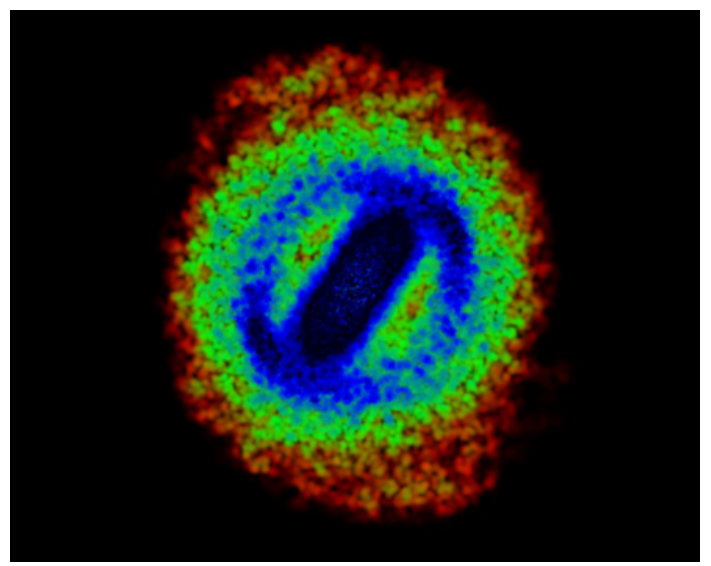

Figure 3. Face-on view of the disc component in a simulation with a strong bar and a ring. Rotation here is clockwise. Note that the density along the ring is not constant, but is in fact somewhat higher in the leading than in the trailing side. If such a structure is also present in our Galaxy, it could result in an estimated bar position angle somewhat larger than the actual value.

The existence of a second bar is not uncommon. About a fourth or a fifth of disc galaxies have both a primary, or main bar and a secondary, or inner bar ([27-29]). Secondary bars have lengths between 0.1 and $1.2 \mathrm{kpc}$, and in relative size they are about $10-15 \%$ of the main bar. These numbers contrast strongly with those found for our Galaxy, where the length of the COBE/DIRBE bar is found to be about $3-3.5 \mathrm{kpc}$ and that of the Long bar about $4 \mathrm{kpc}$. Double bars have also been found in N-body simulations of disc galaxies, with relative lengths that agree well with observations, and not with those of the COBE and Long bar in the MW ([30, 31]). It can thus be excluded that the COBE/DIRBE and the Long bar are a double bar system similar to that seen in external galaxies. They could indeed form a double bar system only if this was very different from those observed in external galaxies. Since it is hazardous to assume that our Galaxy is very different morphologically from external ones, this possibility should be discarded.

So, if the COBE/DIRBE bar and the Long bar do not constitute a double bar system, then what are they? The data give us two important clues on that. First, the ratio of the major- to $z$ - semi-axis of the bar is $\sim 0.3$ for the COBE/DIRBE bar and $\sim 0.026$ for the Long bar, i.e. the Long bar is very thin and the COBE/DIRBE bar is very thick. Second, the ratio of the lengths of these two bars $(\sim 0.8)$ is in good agreement with the ratio of the extent of the $b / p$ to that of the thin outer part of bars found by orbital structure analysis and $N$-body simulations (e.g. [7, 12]). Both these clues point to the fact that there is only one bar in our Galaxy of which the thick inner part (b/p bulge) is the COBE/DIRBE bar and the thin outer part is the Long bar. This bar structure, in good agreement with theory and observations from external galaxies, was first proposed for our Galaxy by [33] and first tested by [23] using their red clump giants measurements.

This would have been a clear and undisputed result, had it not been for the fact that observations argue for the the COBE/DIRBE bar and the Long bar having considerably different position angles, between $15^{\circ}$ and $30^{\circ}$ for the former and around $43^{\circ}$ for the latter. How can this be reconciled with the single bar picture presented above? Let me first say that, due to our location within the Galaxy (see Sect. 1), the estimates for the Galactic bar position angles are much less accurate than the corresponding estimates for external galaxies. Thus Zasowski, Benjamin \& Majewski (poster at this meeting) find the position angle of the Long bar to be between $25^{\circ}$ and $35^{\circ}$, i.e. much closer to that of the COBE/DIRBE bar than the $43^{\circ}$ estimated by $[21-23,25]$. 
A further point to note is that, if the outer isodensity contours of the thin part of the bar are, in the equatorial plane, more rectangular-like than elliptical-like, as is often the case in external galaxies (e.g. [34-36]), the bar position angle will appear to be at larger angles than what it actually is. A final important point is that in many N-body simulations there is often an inner ring and within it a short, leading segment near the end of the bar (see e.g. [32, 37, 38], and Fig. 3 here). In view of all the above comments, the small difference in position angle between the COBE/DIRBE and the Long bar, should not be a major concern.

We are thus led to the conclusion that the COBE/DIRBE and the Long bar are parts of the same bar, and do not constitute two separate components. There is, therefore, good agreement between, on the one hand, the properties of the bar/bulge system in our Galaxy, and, on the other, observations of external galaxies, simulations and orbital structure theory. Such an agreement is of paramount importance, since our Galaxy should not be different from other disc galaxies of the same type.

A more extended version of the discussion presented in sections 2 and 3 can be found in [38]. After my talk, I. Martinez-Valpuesta presented a contribution reaching a very similar result, although in a different way. This work is now published [37].

\section{FURTHER RESULTS ON BARS}

\subsection{Bars formed in disc galaxies with gas and with triaxial haloes}

Although quite extensive, most previous studies of bars rely on at least one of the two following simplifying approximations: That haloes are spherical and that the gaseous content of disc galaxies can be neglected, or at least modelled in an oversimplified way. Both these approximations were abandoned by Athanassoula, Machado \& Rodionov (in prep.), who considered triaxial haloes and a gaseous content of the disc modelled including star formation, feedback and cooling. They find that these new effects considerably influence the bar morphology, strength and length, as well as the gas distribution. In general, haloes evolve towards axisymmetry even if initially very triaxial, or in the presence of a considerable gaseous component. In several previous studies, albeit with spherical haloes and no gas, it was shown that the inner part of the halo becomes prolate, forming what was termed a 'halo bar', or 'dark bar' (e.g. [39-43]). Our study confirmed these results for cases where the initial gaseous content is rather low and the halo either spherical, or mildly triaxial. In the remaining cases, however, we find that the central regions become, on the contrary, more spherical.

\subsection{Bars in a cosmological framework}

Scannapieco \& Athanassoula (in prep.) discuss two examples of the formation of bars in fully cosmological hydrodynamical simulations and compare the resulting bar properties to both those of bars formed in dynamical (idealised) simulations and to those of observed bars. Some of their results can be found in an upcoming paper and show that realistic bars can form naturally in $\Lambda \mathrm{CDM}$ simulations, where host galaxies grow, accrete matter and significantly evolve during the formation and evolution of the bar. More specifically, this work addresses the length and strength of bars and their effect on the radial projected density profiles and on the velocity field.

I thank my collaborators Albert Bosma, Merce Romero-Gomez, Rubens Machado, Sergey Rodionov and Cecilia Scannapieco for interesting and motivating discussions, Jean-Charles Lambert for his help with GLNEMO and the organisers for inviting me to this very interesting meeting. 
EPJ Web of Conferences

\section{References}

[1] K. Sheth et al., ApJ, 675, 1141 (2008)

[2] E. Athanassoula, MNRAS, 358, 1477 (2005a)

[3] G. Contopoulos, T. Papayannopoulos, A\&A, 92, 33 (1980)

[4] E. Athanassoula, O. Bienaymé, L. Martinet, D. Pfenniger, A\&A, 127, 349 (1983)

[5] D. Pfenniger, 1984, 134, 373 (1984)

[6] Ch. Skokos, P.A. Patsis, E. Athanassoula, 2002a, MNRAS, 333, 847 (2002a)

[7] P.A. Patsis, Ch. Skokos, E. Athanassoula, 2002a, MNRAS, 337, 578 (2002a)

[8] R. Lütticke, R.-J. Dettmar, M. Pohlen, A\&A, 362, 435 (2000)

[9] M. Bureau, G. Aronica, E. Athanassoula, R.-J. Dettmar, A. Bosma, K.C. Freeman, MNRAS, 370, 753 (2006)

[10] D. Bettoni, G. Galletta, A\&A, 281, 1 (1994)

[11] A.C. Quillen, L.E. Kuchinski, J.A. Frogel, D.L. DePoy, ApJ, 481, 179 (1997)

[12] E. Athanassoula, R.L. Beaton, MNRAS, 370, 1499 (2006)

[13] J.L. Weiland et al., ApJ, 425, L81 (1994)

[14] E. Dwek, et al., ApJ, 445, 716 (1995)

[15] J. Binney, O. Gerhard, D. Spergel, MNRAS, 288, 365 (1997)

[16] K.Z. Stanek, A. Udalski, M. Szymanski, J. Kaluzny, M. Kubiak, M. Mateo, \& W. Krzeminski, ApJ, 477, 163 (1997)

[17] W. Dehnen, AJ, 119, 800 (2000)

[18] M. López-Corredoira, A. Cabrera-Lavers, O.E. Gerhard, A\&A, 439, 107 (2005)

[19] C. Hamadache, et al., A\&A, 454, 185 (2006)

[20] I. Minchev, J. Nordhaus, \& A.C. Quillen, ApJ, 664, L31 (2007)

[21] P.L. Hammersley, F. Garzón, T.J. Mahoney, M. López-Corredoira, M.A.P. Torres, MNRAS 317, L45 (2000)

[22] R.A. Benjamin et al., ApJ, 630, L149 (2005)

[23] A. Cabrera-Lavers, P.L. Hammersley, C. González-Fernández, M. López-Corredoira, F. Garzón, T.J. Mahoney, A\&A, 465, 825 (2007)

[24] M. López-Corredoira, A. Cabrera-Lavers, T.J. Mahoney, P.L. Hammersley, F. Garzón, C. González-Fernández, AJ, 133, 154 (2007)

[25] Cabrera-Lavers, A., González-Fernández, C., Garzón, F., Hammersley, P. L., \& M. LópezCorredoira, A\&A, 491, 781 (2008)

[26] E. Churchwell, et al., PASP, 121, 213 (2009)

[27] P. Erwin, L. Sparke, AJ, 124, 65 (2002)

[28] S. Laine, I. Shlosman, J.H. Knapen, R.F. Peletier, ApJ, 567, 97 (2002)

[29] P. Erwin, Tumbling, twisting, and winding galaxies: Pattern speeds along the Hubble sequence, E. M. Corsini and V. P. Debattista (eds), Memorie della Societa'Astronomica Italiana, 18, 145 (2011)

[30] C.H. Heller, I. Shlosman, E. Athanassoula, ApJ, 657, L65 (2007)

[31] J. Shen, V.P. Debattista, ApJ, 690, 758 (2009)

[32] E. Athanassoula, A. Misiriotis, MNRAS, 330, 35 (2002)

[33] E. Athanassoula, Mapping the Galaxy and Nearby Galaxies, eds. K. Wada and F. Combes, 47 (Springer, Berlin, 2008)

[34] E. Athanassoula, S. Morin, H. Wozniak, D. Puy, M.J. Pierce, J. Lombard, A. Bosma, MNRAS, 245, 130 (1990)

[35] D.A. Gadotti, Chaos in Astronomy, G. Contopoulos and P. Patsis (eds.), (Springer, Berlin), 159 (2008)

[36] D.A. Gadotti, MNRAS, 415, 3308 (2010)

[37] I. Martínez-Valpuesta, O. Gerhard, ApJL 734, L20 (2011) 
Assembling the Puzzle of the Milky Way

[38] M. Romero-Gómez, E. Athanassoula, T. Antoja, F. Figueras, MNRAS, (2011) 10.1111/j.13652966.2011.19569.x and arXiv:1108.0660

[39] E. Athanassoula, Celestial Mechanics and Dynamical Astronomy, 91, 9 (2005b)

[40] P. Colín, O. Valenzuela, A. Klypin, ApJ, 644, 687 (2006)

[41] I. Berentzen, I. Shlosman, ApJ, 648, 807 (2006)

[42] E. Athanassoula, MNRAS, 377, 1569 (2007)

[43] R. Machado, E. Athanassoula, MNRAS, 406, 2386 (2010) 